\title{
Metastasis of pulmonary adenocarcinoma to the palatine tonsil
}

\author{
LENA ZAUBITZER ${ }^{1}$, NICOLE ROTTER ${ }^{1}$, CHRISTOPH ADERHOLD $^{1}$, TIMO GAISER $^{2}$, FREDERIC JUNGBAUER $^{1}$, \\ BENEDIKT KRAMER $^{1}$, BEATRICE KUHLIN ${ }^{1}$, CARSTEN THORN ${ }^{1}$ and ANNE LAMMERT ${ }^{1}$
}

${ }^{1}$ Department of Otorhinolaryngology Head and Neck Surgery; ${ }^{2}$ Institute of Pathology, University Hospital Mannheim, Medical Faculty Mannheim, University Heidelberg, D-68167 Mannheim, Germany

Received June 8, 2018; Accepted October 16, 2018

DOI: $10.3892 /$ mco.2018.1776

\begin{abstract}
Lung cancer rarely metastasizes to the palatine tonsil. There have only been a few cases that have reported on palatine tonsillar metastases of pulmonary adenocarcinoma. In the majority of these cases the primary diagnosis is a small cell lung cancer. The present case report resents an unusual case of a 66-year-old woman with a multiple metastasized lung adenocarcinoma, which was finally detected by tonsillectomy. The present case report and review of the literature revealed the importance of complete and systematic tumor staging, including the examination of the oral cavity in case of lung cancer and that the evident tentative diagnosis is not always the right one.
\end{abstract}

\section{Introduction}

Adenocarcinoma is the most common histological type of primary lung cancer and shows an increasing incidence all over the world (1). Lung cancer most frequently metastasizes lymphogenous to regional lymph nodes and haematogenous to extra-pulmonary organs like the brain, bones, liver, and adrenal glands (2). Metastases from lung cancer to the palatine tonsils are very uncommon (3).

Regarding all malignant tonsillar tumors, only $0.8 \%$ are distant metastases from tumors of distant origin (4). For tonsillar metastases that originate from the lung, small cell lung cancer is more frequent than pulmonary adenocarcinoma (4).

Following we report on a rare case of distant metastasis of a primary adenocarcinoma of the lung to the palatine tonsil and present a review of the literature.

Correspondence to: Dr Anne Lammert, Department of Otorhinolaryngology Head and Neck Surgery, University Hospital Mannheim, Medical Faculty Mannheim, University Heidelberg, Theodor-Kutzer-Ufer 1-3, D-68167 Mannheim, Germany

E-mail: anne.lammert@umm.de

Key words: tonsillar metastasis, pulmonary adenocarcinoma, palatine tonsils

\section{Case report}

Clinical findings. A 66-year-old female patient was referred from an ENT-practitioner to our ENT clinic because of a right-sided cervical lymphadenopathy and an asymmetry of the palatine tonsils. After treatment with oral antibiotics (Amoxicillin) for 7 days without recovery she was sent to an ENT specialist by her general practitioner.

Anamnesis revealed an intended loss of body weight of approximately $20 \%$ during the last two years. She had a hoarse voice but no dysphagia and no dyspnoea. Moreover she was a former smoker with about 40 pack years, but stopped 10 years ago. The physical examination showed asymmetrical palatine tonsils with a bigger and indurated right palatine tonsil. Enlarged coalescent and firm lymph nodes were palpable in level I to IV of the right side of her neck. The sonography showed multiple enlarged lymph nodes with a maximal diameter of two centimeters.

After radiological staging a panendoscopy was performed. The fiberoptic bronchoscopy showed an epithelial lesion on the level of the right medial main bronchus, from which a biopsy was taken. The examination of the oropharynx under general anaesthesia revealed an indurated mass arising from the right palatine tonsil, reaching the uvula and the glossotonsillar fossa. Two additional biopsies were taken, one from the right palatine tonsil and the other from the glossotonsillar fossa. Written informed consent was obtained from the patient.

Histopathological findings. While biopsies of the glossotonsillar fossa showed regular histology, a diffuse infiltrative growing, poorly differentiated adenocarcinoma with vascular invasion was present in the right palatine tonsil and in the right medial main bronchus (Fig. 1A and B). Immunohistochemistry results were equal in both locations and the tumor cells expressed cytokeratin 7 and TTF1 (Fig. 1C and D) while immunohistochemistry against CK20, p63, p40 and p16 showed no staining. In sum, the diagnosis of a metastatic pulmonary adenocarcinoma was established based on these findings. Molecular pathological work-up further revealed no $E G F R$ or $B R A F$ mutation. There was no PDL-1 expression nor any staining positivity for ALK or ROS1.

Radiological findings. Neck and chest CT-scan confirmed the diagnosis of a lymphogenous and haematogenous metastasized carcinoma of the right lung. Chest CT-scan revealed a tumor 
mass in the region of the right lower hilus (Fig. 2A). It showed enlarged lymph nodes on the right side of the neck with a maximum diameter of $2 \mathrm{~cm}$ (Fig. 2B) and enlarged axillar lymph nodes on the left side. Moreover it revealed mediastinal lymph nodes suspicious for metastases and presternal and adrenal gland metastases.

Cranial MRI-scan showed five neurocranial lesions (one right frontal, two left temporal, one parietooccipital and parietal), which were suggestive of brain metastases.

MRI findings of the abdomen revealed contrast-enhanced lesions in both adrenal glands. The scans showed some periportal and peristernal lesions, which were in line with lymph node metastasis.

Initiation of therapy. Upon the recommendation of a multidisciplinary tumor board the patient received a gamma knife stereotaxic radiosurgery of the five brain metastases each with 22 Gy per single dose. During the inpatient stay in the context of the radiation, skin metastases at her right thigh were detected.

The radiotherapy was followed by a palliative chemotherapy with cisplatin and pemetrexed. At this juncture the patient already received five of six planned bouts of chemotherapy. The last cranial MRI-scan correlated with the pattern of a stable disease.

\section{Discussion}

Bronchial carcinoma are classified into different histological subtypes. One of the most important classifications differs between small cell lung carcinoma (SCLC) and non-small cell lung carcinoma (NSCLC) (5). Pulmonary adenocarcinoma belongs to the group of NSCLC (6). With about $38 \%$ of all lung cancers adenocarcinomas are the most prevalent type (7). Adenocarcinoma of the lung can be spread lymphogenously to lymph nodes or haematogenously via blood vessels to extra-pulmonary organs like the adrenal glands, brain and bones. The different histological subtypes of lung cancer show a different organ preference for the metastatic spread. Brain metastases originate in most cases from small cell lung cancer and adenocarcinoma, metastases placed in the liver often spring from small cell lung cancer but not from adenocarcinoma (8).

Carcinomas of the oropharynx account over $10 \%$ of all head neck cancers (9). About $70-80 \%$ of all primary oropharynx carcinoma are located in the tonsillar complex (10). Over $80 \%$ of palatine tonsillar carcinoma are squamous cell carcinoma followed by lymphoma (12\%). Other histological cancer subtypes than squamous cell carcinoma represent $\sim 4 \%$. Adenocarcinoma originating from the lung occur in $<1 \%$ (11).

Between all palatine tonsillar neoplasms, metastases are very rare. In a study with 1535 cases of malignant palatine tonsillar tumors only $0.8 \%$ were metastases from an extra-tonsillar primary (12). Case reports can be found about tonsillar metastases originating from breast cancer, colorectal carcinoma, seminoma, gastric carcinoma, renal cell carcinoma and melanoma $(4,9)$. In another study, in 12 of 76 cases of palatine tonsillar metastases the primary tumor is localized in the lung. 10 of these 12 pulmonary carcinomas were small cell lung cancer and only the two other cases were lung adenocarcinoma (4).
There are different theories discussed about the way of cancer cell spreading. Retrograde lymphatic as well as haematogenous cell movement to the palatine tonsils are possible (13). It is assumed that cancer cells spread most frequently haematogenously via blood vessels to the palatine tonsils (4). The lymphatic way of cell spreading is less likely because there are no afferent lymphatic vessels leading to the palatine tonsils. But retrograde cell spreading seems to be possible if there are metastases in the cervical lymph nodes leading to an inversion of the fluid flow in the lymphatic vessels (4). In our case there were metastases in the right cervical lymph nodes, so that a retrograde lymphatic movement of cancer cells is a possible explanation for the metastasis. The retrograde flow can be explained by an invasion of carcinoma cells to a lymphatic vessel, which is followed by a higher down-stream pressure in the lymphatic vessel. Normally bileaflet valves inhibit retrograde lymphatic flow, but as a consequence of adhesion of tumor cells their function can become defective (4).

Another theory discussed for palatine tonsillar metastases of lung cancer is the direct implantation of carcinoma cells to the tonsils during bronchoscopy (4). In our reported case a direct implantation is ruled out because the patient did not have any diagnostic intervention up to the presumption diagnosis.

Most reported cases of palatine tonsillar metastases of lung cancer are about small cell lung cancer. But in our case immunohistochemical analyses of the tumor cells were positive for pan cytokeratin, CK7 and TTF1 and negative for CK20, p63, p40 and p16. These results are in favour for a poorly differentiated pulmonary adenocarcinoma. Further analyses for $E G F R$ mutation and $B R A F$ mutation were negative. EGFR mutation analyse should be part of the diagnostic, because in case of a mutation, a therapy with small-molecule receptor tyrosine kinase inhibitors may be possible (14). CEA serum level is a prognostic and predictive marker for risk of recurrence and death in NSCLC. Increased CEA serum values measured before treatment are considered as to be a prognostic marker for NSCLC (15). Moreover high CEA values seem to be associated with an increased risk of brain metastasis and a poor prognosis. Particularly in patients with adenocarcinoma and CEA $>40 \mathrm{ng} / \mathrm{ml}$ at diagnosis a higher risk of development of brain metastasis was ascertained (16). In the described case the plasma CEA level at diagnosis was $51 \mathrm{mg} / 1(51,000 \mathrm{ng} / \mathrm{ml})$.

In our case an initial differential diagnosis of the palatine tonsilliar metastasis of the bronchial carcinoma was a T2N2bM1 oropharyngeal cancer of the right palatine tonsil. In consideration of the multiple metastases and the palliative situation of the patient, she received a gamma knife stereotaxic radiosurgery, followed by chemotherapy with cisplatin and pemetrexed with palliative intention. Because of the absence of oncogenetic drivers and the absent PD-L1 expression, a targeted therapy was not possible.

Different types of therapy are reported in case of metastases to the palatine tonsils. Therapeutic interventions described include surgery, chemotherapy, radiotherapy and chemoradiotherapy (17). A recommendation for therapeutic proceeding in case of palatine tonsillar metastasis does not exist. Recommendations about therapy should be made inter-individual and in consideration of the patient's general condition and tumor stage in an interdisciplinary setting. 
A

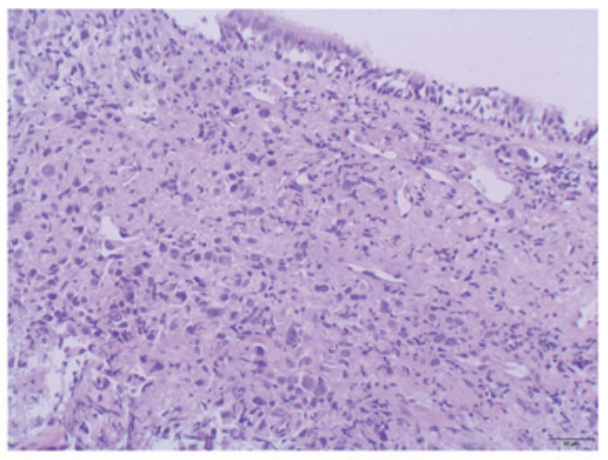

C

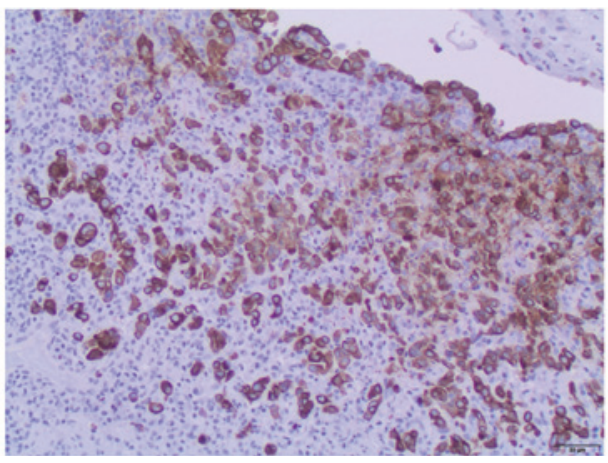

B

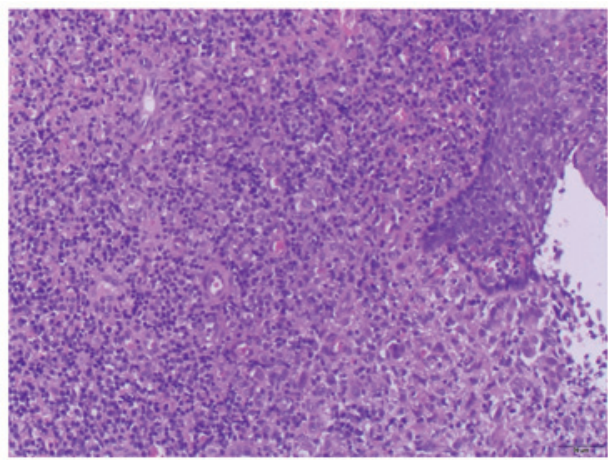

D

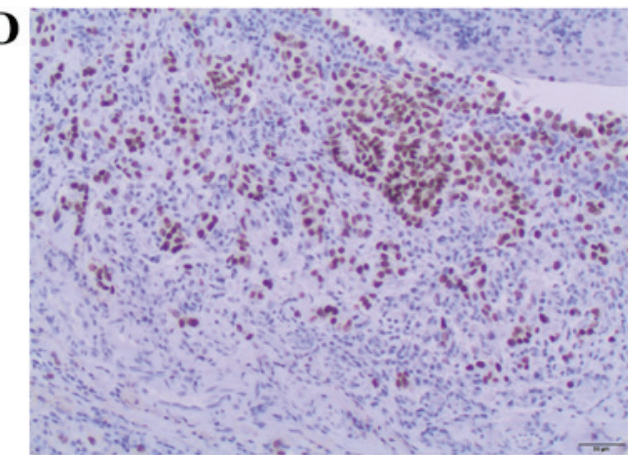

Figure 1. Histopathological results. (A) Respiratory columnar epithelium of the right medial main bronchus undermined by a poorly differentiated carcinoma with lymphovascular invasion hematoxylin and eosin staining; magnification, x100). (B) Stratified squamous epithelium of the right palatine tonsil presenting a histological identical undermining cancerous infiltrate (magnification, x100). (C) Cytokeratin 7 and (D) thyroid transcription factor-1 positivity establishes the diagnosis of a metastatic pulmonary adenocarcinoma (magnification, $\mathrm{x} 100$ ).
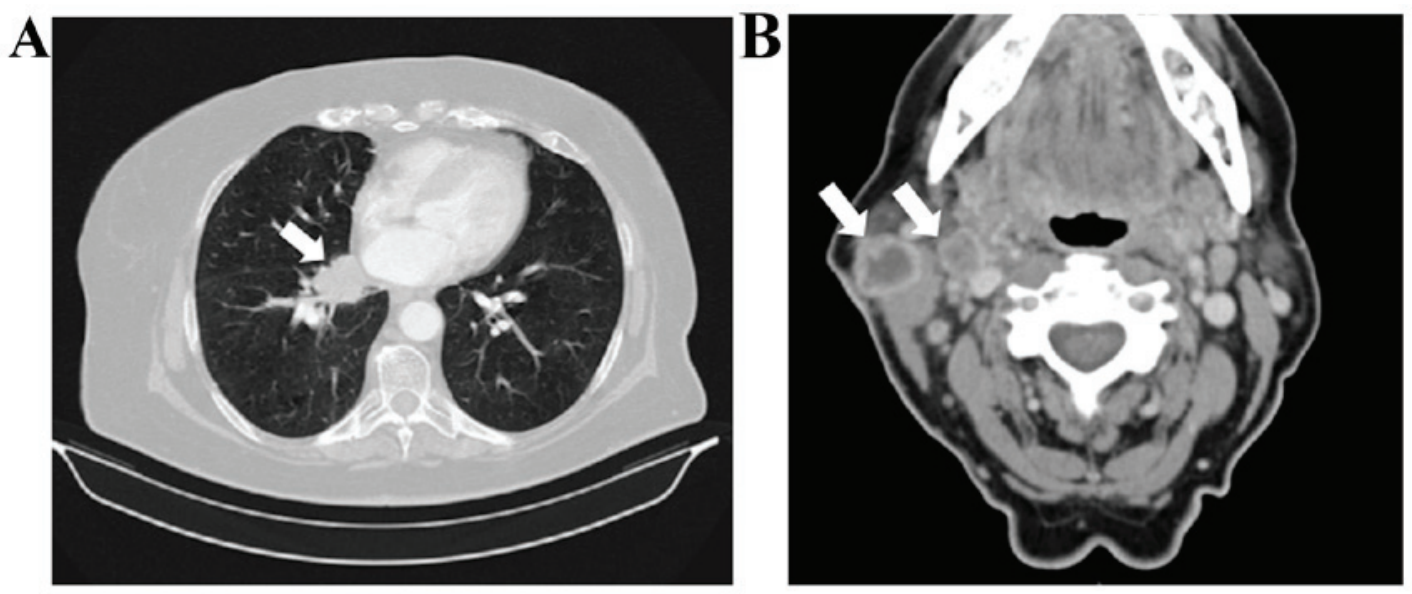

Figure 2. Radiological results. (A) Neck and chest computed tomography-scan revealed a tumor mass in the region of the right lower hilus (white arrow) (B) and enlarged lymph nodes on the right side of the neck with a maximum diameter of $2 \mathrm{~cm}$ (white arrows).

In conclusion, metastases of lung cell adenocarcinoma to the palatine tonsils are very rare, nevertheless this unusual case should be kept in mind during the physical examination of patients with lung cancer.

\section{Acknowledgements}

Not applicable.

\section{Funding}

No funding was received.

\section{Availability of data and materials}

The datasets used and/or analyzed during the current study are available from the corresponding author on reasonable request.

\section{Authors' contributions}

ZL performed the patient procedures, and was involved in patient diagnosis and therapy. LA was also involved in patient therapy. ZL and LA critically revised the manuscript for important intellectual content. RN, AC, JF, KB, KB and $\mathrm{TC}$ contributed ideas and assisted with the completion of the 
manuscript, revising it critically for important intellectual content. GT performed the histological examination of the patient's materials. All authors read and approved the final manuscript.

\section{Ethics approval and consent to participate}

Written informed consent for the use of tissues was obtained from the patient.

\section{Patient consent for publication}

Written informed consent was obtained from the patient.

\section{Competing interests}

The authors declare that they have no competing interests.

\section{References}

1. Nakamura H and Saji H: Worldwide trend of increasing primary adenocarcinoma of the lung. Surg Today 44: 1004-1012, 2014.

2. Yang CJ, Hwang JJ, Kang WY, Chong IW, Wang TH, Sheu CC, Tsai JR and Huang MS: Gastro-intestinal metastasis of primary lung carcinoma: Clinical presentations and outcome. Lung Cancer 54: 319-323, 2006.

3. Arroyo HH, Takehara J, Ogawa AI, Frizzarini R, Imamura R and Paula HM: Small cell lung carcinoma metastasis to palatine tonsils. Braz J Otorhinolaryngol 79: 645, 2013 (In English and Portuguese).

4. Tajima S and Koda K: Palatine tonsillar metastasis of a small pulmonary adenocarcinoma showing an invasive micropapillary carcinoma pattern and Pagetoid spread at the tonsil: A case suggesting retrograde lymphatic metastasis from bulky lymph node metastases of the neck. Int J Clin Exp Pathol 8: 13601-13653, 2015.

5. Travis WD: Pathology of lung cancer. Clin Chest Med 32 669-692, 2011
6. Travis WD, Brambilla E and Riely GJ: New pathologic classification of lung cancer: Relevance for clinical practice and clinical trials. J Clin Oncol 31: 992-1001, 2013.

7. Travis WD: Classification of lung cancer. Semin Roentgenol 46: 178-186, 2011.

8. Popper HH: Progression and metastasis of lung cancer. Cancer Metastasis Rev 35: 75-91, 2016.

9. Sommer F, Rettinger G and Kastl K: Unilateral degeneration of tissue of the tonsil in a patient with adeno-carcinoma of the lung. Laryngorhinootologie 90: 30-31, 2011 (In German).

10. Skinner HD, Holsinger FC and Beadle BM: Oropharynx cancer. Curr Probl Cancer 36: 334-415, 2012.

11. Golas SM: Trends in palatine tonsillar cancer incidence and mortality rates in the United States. Community Dent Oral Epidemiol 35: 98-108, 2007.

12. Hyams VJ: Differential diagnosis of neoplasia of the palatine tonsil. Clin Otolaryngol Allied Sci 3: 117-126, 1978.

13. D'Antonio C, Lombardini A, Onesti CE, Falcone R, Romiti A, Lombardi M, Lauro S and Marchetti P: A rare case of palatin tonsillar metastasis from small cell lung cancer. Transl Lung Cancer Res 5: 709-711, 2016.

14. Travis WD, Brambilla E, Noguchi M, Nicholson AG, Geisinger KR, Yatabe Y, Beer DG, Powell CA, Riely GJ, Van Schil PE, et al: International association for the study of lung cancer/American thoracic society/european respiratory society international multidisciplinary classification of lung adenocarcinoma. J Thorac Oncol 6: 244-285, 2011.

15. Grunnet M and Sorensen JB: Carcinoembryonic antigen (CEA) as tumor marker in lung cancer. Lung Cancer 76: 138-143, 2012.

16. Arrieta O, Saavedra-Perez D, Kuri R, Aviles-Salas A, Martinez L, Mendoza-Posada D, Castillo P, Astorga A, Guzman E and De la Garza J: Brain metastasis development and poor survival associated with carcinoembryonic antigen (CEA) level in advanced non-small cell lung cancer: A prospective analysis. BMC Cancer 9: 119, 2009.

17. Unsal M, Kutlar G, Sullu Y and Yurtlu S: Tonsillar metastasis of small cell lung carcinoma. Clin Respir J 10: 681-683, 2016.

(i) 1 This work is licensed under a Creative Commons Attribution-NonCommercial-NoDerivatives 4.0 International (CC BY-NC-ND 4.0) License. 